ИЗВЕСТИЯ АКАДЕМИИ НАУК ЭСТОНСКОИ ССР. ФИЗИКА * МАТЕМАТИКА PROCEEDINGS OF THE ACADEMY OF SCIENCES OF THE ESTONIAN SSR. PHYSICS * MATHEMATICS

1987, 36, 4

УДК $535.34: 537.288 .5$

Я. КИKAC

\title{
ПОВЫШЕНИЕ ОРИЕНТАЦИОННОЙ СЕЛЕКТИВНОСТИ ФОТОВЫЖИГАНИЯ СПЕКТРАЛЬНЫХ ПРОВАЛОВ ВО ВРАЩАЮЩЕМСЯ ЭЛЕКТРИЧЕСКОМ ПОЛЕ
}

J. KIKAS. SPEKTRITE FOTOSALKAMISE ORIENTATSIOONILISE SELEKTIIVSUSE KASV POORLEVAS ELEKTRIVALJAS

J. KIKAS. ENHANCEMENT OF THE ORIENTATIONAL SELECTIVITY OF SPECTRAL HOLEBURNING IN A ROTATING ELECTRIC FIELD

(Представил К. К. Ребане)

1. Фотовыжигание спектральных провалов (ФСП) $\left[{ }^{1,2}\right]$ позволяет осуществить высокодобротную селекцию примесей по частоте бесфононного перехода. В разупорядоченных матрицах (стеклах) степень селекции может достигать $10^{5}-10^{6}$ (отношение ширин неоднородной спектральной полосы и однородной чисто электронной линии). Пред-

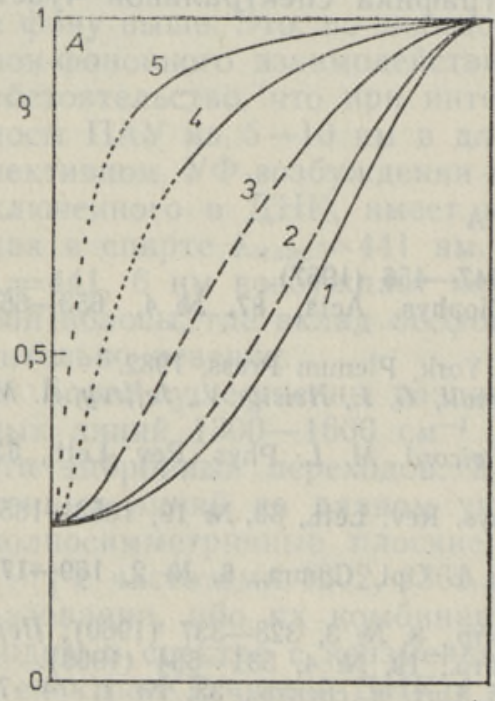

0

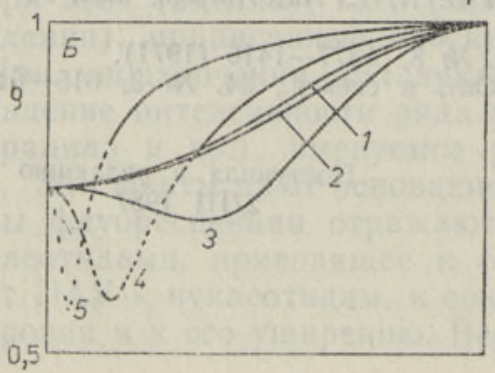

ставляет интерес использование такой высокой добротности для улучшения селективности и по другим параметрам, например, по ориентациям примесей в ориентационно неоднородных системах. Ниже показано, что повышение ориентационной селективности ФСП может быть достигнуто с помощью приложения вращающегося электрического поля.

2. Рассмотрим процесс ФСП в основной модели $\left(\left[{ }^{3}\right]\right.$, с. 237$)$. Дополнительно предполагаем следующее:

2.1. Однородный спектр поглощения содержит только лоренцеву бесфононную линию с шириной $\Gamma$.

A. Угловое распределение резонансных $(\omega=$ $=v_{0}$ ) центров при времени выжигания $\sqrt{2 \pi} / I \eta \sigma$ и значения $E d / \Gamma=0$ (кривая I); 0,5 (2); 5 (3); 50 (4) н 500 (5). Приведено меридиональное сечение $(0 \leqslant \theta \leqslant \pi / 2, \varphi=$ const) двумерного ориентационного распределення $\varrho(\omega, \Theta, \varphi, t)$, нормированное на начальное значение $\mathrm{Q}=\mathrm{Q}(\omega, \Theta, \varphi, t) / \mathrm{\varrho}(\omega, \Theta, \varphi, 0)$.

b. То же для неполностью резонансных центров $\left(\left|\omega-v_{0}\right|=\Gamma\right)$. 
2.2. Актуальный переход является дипольным и линейно поляризованным. Распределение направлений поглощающих диполей изотропное.

2.3. На переходе проявляется линейный Штарк-эффект, при этом разница дипольных моментов основного и возбужденного состояний $\overleftrightarrow{d}$ колинеарна моменту перехода.

2.4. Система возбуждается вертикально поляризованным монохроматическим светом частотой $v_{0}$ и интенсивностью $I$.

Легко показать, что изменяющаяся в процессе выжигания частотноориентационная функция неоднородного распределения (ФНР) имеет вид (см. также $\left[{ }^{4}\right]$ )

$$
\varrho(\omega, \Theta, \varphi, t)=\varrho(\omega, \Theta, \varphi, 0) \exp \left\{-\frac{I t \eta \sigma \Gamma \cos ^{2} \Theta}{2 \pi\left[(\Gamma / 2)^{2}+\left(v_{0}-\omega\right)^{2}\right]}\right\},
$$

где $t-$ время и $\eta-$ квантовый выход выжигания, $\sigma$ - интегральное сечение поглощения, $\omega-$ частота бесфононного перехода. $\Theta$ и $\varphi-$ соответственно полярный и азимутальный углы, определяющие ориентацию диполя поглощения. Система сферических координат выбрана таким образом, что $\Theta$ изменяется от 0 до $\pi$ (угол между вертикальным направлением и направлением диполя поглощения). «Ориентационный провал» определяется фактически множителем $\cos ^{2} \Theta$ в экспоненте. Из рисунка, $A$ видно (кривая 1 ), что селективность выжигания по углу $\Theta$ невысока.

3. Пусть теперь одновременно с монохроматическим светом к образцу приложено электрическое поле напряженности $E$, вектор которого вращается в горизонтальной плоскости с угловой скоростью $\Omega\left(t \gg \Omega^{-1} \gg \Gamma^{-1}\right)$. Тогда в формуле (1) частоту $\omega$ надо заменить на измененную из-за Штарк-эффекта

$$
\omega \rightarrow \omega+E d \sin \Theta \sin \left(\Omega t^{\prime}+\varphi_{0}-\varphi\right)
$$

и произвести в экспоненте усреднение по периоду вращения $\Delta t^{\prime}=$ $=2 \pi / \Omega$. В (2) $d=|\vec{d}|$. В результате получим

$$
\begin{gathered}
\varrho(\omega, \Theta, \varphi, t)=\varrho(\omega, \Theta, \varphi, 0) \times \\
\times \exp \left\{-I \eta \eta \sigma \frac{\cos ^{2} \Theta}{\pi \sqrt{2}}\left\{\frac{D+\left[D^{2}+\Gamma^{2}\left(v_{0}-\omega\right)^{2}\right]^{1 / 2}}{D^{2}+\Gamma^{2}\left(v_{0}-\omega\right)^{2}}\right\}^{1 / 2}\right\},
\end{gathered}
$$

где применено обозначение

$$
D=E^{2} d^{2} \sin ^{2} \theta+(\Gamma / 2)^{2}-\left(v_{0}-\omega\right)^{2} .
$$

Для резонансных центров

$$
\varrho\left(v_{0}, \Theta, \varphi, t\right)=\varrho\left(v_{0}, \Theta, \varphi, 0\right) \exp \left\{-\pi^{-1} I \eta t \sigma \cos ^{2} \Theta\left[(\Gamma / 2)^{2}+E^{2} d^{2} \sin ^{2} \Theta\right]^{-1 / 2}\right\} \text {. }
$$

Относительное изменение угловой ФНР для этого случая изображено на рисунке, $A$ для разных значений $E d$, где видно повышение ориентационной селективности с ростом $E d$. Физическая причина роста ориентационной селективности довольно простая. Только центры с разницей дипольных моментов $\vec{d}$, перпендикулярной плоскости вращения электрического поля, не испытывают штарковского сдвига частоты перехода. Остальные же центры на более или менее длительное время выводятся из резонанса с монохроматическим светом, вследствие чего понижается усредненная по периоду $\Delta t^{\prime}$ эффективность выжигания. При малых временах выжигания и большом отношении $E d / \Gamma$ получаем 
из формулы (4) следующее выражение для полуширины ориентационного провала $\Theta_{1 / 2}$ (угол, при котором доля выжженных центров в два раза меньше по сравнению с $\Theta=0)$ :

$$
\Theta_{1 / 2}=(\sqrt{3} / 2) \Gamma / E d \approx 0,87 \Gamma / E d .
$$

Вместе с тем для неполностью резонансных центров наблюдается отличающаяся картина (рисунок, Б). Наиболее эффективно выжигаются центры, дипольный момент которых находится под определенным углом относительно поляризации света. Это связано с синусоидальным законом модуляции частоты (2).

4. Приведем некоторые оценки для реальных систем. Влияние электрического поля на спектральные провалы экспериментально исследовалось в ряде работ $\left[{ }^{5-9}\right]$. По данным $\left[{ }^{8,9}\right]$, для молекулы хлорина в матрице поливинилбутирала при $T=2 \mathrm{~K} \Gamma \approx 0,02 \mathrm{~cm}^{-1}$ и пробойное напряжение матрицы составляет $5 \cdot 10^{5} \mathrm{~B} \mathrm{~cm}^{-1}$. Учитывая полученное в $\left.{ }^{8}\right]$ значение $d=0,25$ Дебая, получаем $E d / \Gamma \leqslant 100$, т. е. ситуацию, соответствующую кривым 4 на рисунке и являющуюся реально достижимой. Следует, однако, отметить, что мы не знаем эффективного способа для регистрации суженных предложенным образом ориентационных провалов. При обычных методах регистрации суженные согласно (3), (4) ориентационные провалы свертываются с функциями типа $\cos ^{2} \Theta$, что маскирует достигнутое сужение.

Автор благодарен К. К. Ребане за обсуждение и П. Труусалу за помощь в численном расчете.

Л И ТЕ Р А Т У Р А

1. Гороховский А. А., Каарли Р. К., Ребане Л. А. Письма в ЖЭТФ, 20, № 7, 474478 (1974).

2. Kharlamov, B. M., Personov, R. I., Bykovskaya, L. A. Opt. Commun., 12, № 2, 191-193 (1974).

3. Rebane, L., Gorokhovskii, A., Kikas, J. Appl. Phys. B, 29, № 2, 235-250 (1982).

4. Харламов Б. М., Улицкий Н. Н. Препринт ИС ӒН СССР, № 12. Троицк, 1986.

5. Marchetti, A. P., Scozzafawa, M., Young, R. H. Chem. Phys. Lett., 51, № 3, 424-426 (1977).

6. Самойленко В. Д., Разумова Н. В., Персонов Р. И. Опт. и спектр., 52, № 4, $580-582$ (1982).

7. Bogner, U., Schätz, P., Seel, R., Maier M. Chem. Phys. Lett., 102, № 2/3, 267-271 (1983).

8. Burkhalter, F. A., Suter, G. W., Wild, U. P., Samoilenko, V. D., Rasumova, N. V., Personov, R. I. Chem. Phys. Lett., 94, № 5, 483-487 (1983).

9. Wild, U. P., Bucher, S. E., Burkhalter, F. A. Appl. Opt., 24, № 10, 1526-1530 (1985).

Институт физики

Академии наук Эстонской ССР
Поступила в редакцию 12/III 1987 